\title{
Halo K-Giant Stars from LAMOST: Kinematics and Galactic Mass Estimate
}

\author{
Sarah A. Bird \\ Shanghai Astronomical Observatory, 80 Nandan Road, Shanghai 200030, China \\ email: sarahbird@shao.ac.cn
}

\begin{abstract}
We analyze line-of-sight velocities of over 3000 halo K-giant stars from the second data release of the spectral survey LAMOST (Zhao et al. 2012). We find a nearly constant velocity dispersion profile, with no large dips or peaks, in a Galactocentric radial range of $10-30 \mathrm{kpc}$, in accord with earlier analyses (Battaglia et al. 2005, 2006; Xue et al. 2008, 2014) (see Fig. 1). Previous studies of halo star radial velocity dispersions in a reference frame centered on the Galactic Center have detected dips within this radial range (Sommer-Larsen et al. 1994; Kafle et al. 2012, 2014). We use the stars to make estimates of the enclosed mass out to $40 \mathrm{kpc}$ from the Galactic Center using the method of Evans et al. (2011). Tens of thousands of such stars are expected to become available to this analysis by the end of the five-year survey.
\end{abstract}

Keywords. Galaxy: halo — stars: individual (K giants) — stars: kinematics and dynamics

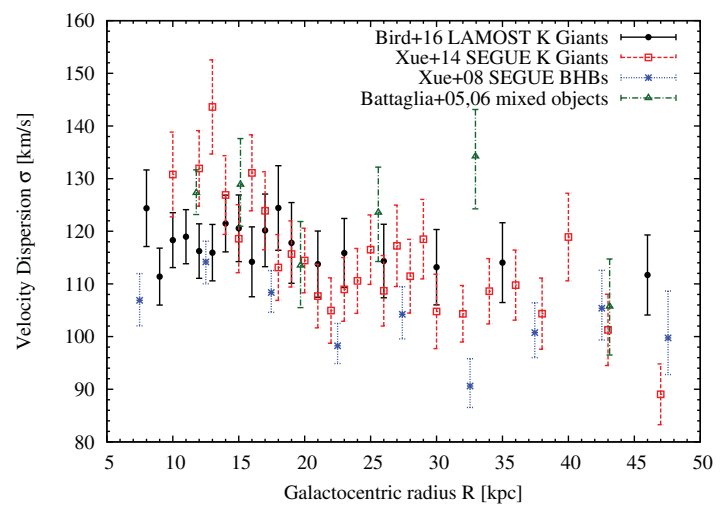

Figure 1. Comparison of line-of-sight halo velocity dispersions from Bird et al. (2016) LAMOST K giants, Xue et al. (2014) SEGUE K giants, Xue et al. (2008) SEGUE blue horizontal branch stars (BHBs), and Battaglia et al. (2005, 2006) mixed objects as a function of Galactocentric radius in spherical coordinates. The profiles follow a flattened distribution.

\section{References}

Battaglia, G., Helmi, A., Morrison, H., et al. 2005, MNRAS, 364, 433

-. 2006, MNRAS, 370, 1055

Bird, S. A., Liu, C., Shen, J., \& Xue, X. X. 2016, in preparation

Evans, N. W., An, J., \& Deason, A. J. 2011, ApJ, 730, L26

Kafle, P. R., Sharma, S., Lewis, G. F., \& Bland-Hawthorn, J. 2012, ApJ, 761, 98

一. 2014, ApJ, 794, 59

Sommer-Larsen, J., Flynn, C., \& Christensen, P. R. 1994, MNRAS, 271, 94

Xue, X. X., Rix, H. W., Zhao, G., et al. 2008, ApJ, 684, 1143

Xue, X.-X., Ma, Z., Rix, H.-W., et al. 2014, ApJ, 784, 170

Zhao, G., Zhao, Y.-H., Chu, Y.-Q., Jing, Y.-P., \& Deng, L.-C. 2012, RAA, 12, 723 